\title{
Performance Evaluation of LTE Network USING MAXIMUM FLOW ALGORITHM
}

\author{
Bir Bahadur Khatri ${ }^{1}$, Bulbul Ahammad ${ }^{1}$, Md. Mezbahul Islam $^{2}$, Rahmina Rubaiat $^{2}$ \\ and Md. Imdadul Islam ${ }^{1}$ \\ ${ }^{1}$ Department of Computer Science and Engineering, Jahangirnagar University, \\ Savar, Dhaka, Bangladesh \\ ${ }^{2}$ Department of Computer Science and Engineering, MBSTU, Tangail, Bangladesh
}

\begin{abstract}
In this paper, we propose a new traffic flow model of the Long Term Evaluation (LTE) network for the Evolved Universal Terrestrial Radio Access Network (E-UTRAN). Here only one Evolve Node B (eNB) nearest to the Mobility Management Entity (MME) and Serving Gateway (S-GW) will use the S1 link to bridge the E-UTRAN and Evolved Packet Core (EPC). All the eNBs of a tracking area will be connected to each other by the X2 link. Determination of capacity of a links of such a network is a challenging job since each node offers its own traffic and at the same time conveys traffic of other nodes. In this paper, we apply maximum flow algorithm including superposition theorem to solve the traffic flow of radio network. Using the total flow per subcarrier, a new traffic model is also developed in the paper. The relation among the traffic parameters: 'blocking probability', 'offered traffic', 'instantaneous capacity', 'average holding time', and 'number of users' are shown graphically under both QPSK and 16-QAM. The concept of the network will be helpful to improve the SINR of the received signal ofeNBslocated long distance relative to $M M E / S-G W$.
\end{abstract}

\section{KEYWORDS}

Aggregate offered traffic, blocking probability, traffic channel, weighted graph and RB.

\section{INTRODUCTION}

Traffic analysis of the LTE network is found in recent research works and few of them which are relevant to the paper is considered here. In [1] analytical model of LTE traffic is shown based on the Markov chain then the traffic model is combined with fading parameters (Rayleigh fading with MIMO) of a wireless link. The probability of forced termination and blocking of calls are plotted against call arrival rate and the number of channels. A similar analysis is found in [2], where $\mathrm{M} / \mathrm{M} / \mathrm{m} / \mathrm{m} / \mathrm{c}$ state transition is used to measure the blocking probability of the LTE network. An algorithm called, 'Extended Erlang B formula' is proposed to evaluate the performance of the network and final 'blocking probability' is plotted against the number of iteration of the algorithm. In [3] soft frequency reuse (SFR) performance of LTE is done by simulation, where the throughput of a cell is plotted against traffic load and cell radius. In [4] authors compare different algorithms pertinent to prioritizing traffic on LTE mobile network. Four traffic parameters: throughput, delay, packet delivery ratio and packet loss ratio are considered to compare the Quality of Service (QoS) of the network and results are shown graphically. In [5] only one traffic parameter called jitter (delay difference) is related with resource block (RB) assignment of LTE network under real time multimedia traffic. In performance evaluation they consider High Definition (HD) video stream of uplink (UE to eNB) using a simulation under Network Simulator (NS3). In [6] authors mainly focus on growth in 
mobile data traffic and enhancement of capacity of LTE network. Application of unlicensed spectrum in LTE network is considered as the best way to increase the data rate of WAN. The pros and cons of existing carried traffic improvement methods: coexistence between LTE-U and Wi-Fi, Dynamic Channel Selection, Listen Before Talk (LBT) traffic, Traffic Offloading, Resource Allocation etc. are discussed with some future issue. In [7] authors proposed traffic model of LTE-WLAN network. The state transition chain of Bianchi model is modified, where the probability of idle and busy state of the network is applied in a different way. Taking typical traffic and wireless link parameters, authors plot the probability of 'transmission of at least one node', 'successful transmission' and 'normalized throughput' against the 'number of nodes'. The impact of size of the 'minimum backoff window' is applied on the graphs of result section, where analytical results are varied by simulation and authors claim high confidence level.

In this paper, we relate the traffic model of LTE with the maximum flow algorithm. Therefore, some works on the maximum flow algorithm and their applications are mentioned in this section. The Ford Fulkerson and Edmonds-Karp algorithm are widely used in the determination of the maximum flow of a system like a computer/communication network, traffic flow, or fluid flow. The solution of the algorithm using C\# is available in [8] with its complexity. In [9] authors propose an incremental maximum flow algorithm, where augmenting routing tree is used to reduce the time complexity of the algorithm. The algorithm is applicable in evaluating the capacity of a dynamic network. Another analysis is found in [10], where maximum flow is determined based on a new technique of augmenting path called draining algorithm. The algorithm works better for a near-saturated network but shows computational complexity like a conventional augmenting path for the case of regular flow networks.

Analysing all the papers of this section we found three research gaps:(1) none of above papers deals with accumulation of data traffic of a tracking area of E-UTRAN to an eNB(as a sink) which is nearest to MME/S-GW then high capacity link between that eNB and EPC will carry the combined traffic flow (2) the maximum flow algorithm is applied for the first time in the EUTRAN region of LTE network (3) the maximum flow algorithm is also modified, where superposition theorem is included with Ford-Fulkerson Algorithm of [11].If the nearest eNB fails then the second nearest eNB will act as the sink unlike the Radio Network Controller (RNC) of 3G.Objective of above modifications of existing LTE network is to improve the SINR of wireless link between eNB and EPC.In this paper three parameters: simultaneous RB of LTE (as traffic channel), the modulation scheme (QPSK and 16-QAM used in LTE), and Erlangs traffic model are combined with aid of maximum flow algorithm to measure the performance of the network.

The rest of the paper is organized as section 2 deals with the proposed radio network of LTE, its traffic model, and the modified maximum flow algorithm, section 3 provides results based on a theoretical analysis of section 2 and section 4 concludes the entire analysis.

\section{SYSTEM MODEL}

This section gives the theoretical analysis of the proposed traffic model and steps of the maximum flow algorithm (combination with superposition theorem) in the context of the proposed radio network. To make the idea clear, we first introduce the physical architecture of the conventional LTE network and the proposed radio network.

\subsection{LTE Network}

LTE network has four important features that make the breakthrough of the previous 3G. The features are femtocell deployment, OFDMA-based physical layer access, MIMO, and elimination of radio network controller (RNC) or base station controller (BSC) of the previous generation. To 
under standard the traffic model of the paper we first introduce the physical architecture of LTEA as shown in fig.1, which mainly consists of two parts: E-UTRAN (radio network) and EPC (core switching part). In E-UTRAN, the eNBs are mutualy connected by the X2 link and to EPC by $\mathrm{S} 1$ link as found in [12-13]. The outdoor traffic is routed through macro eNB and EPC but indoor traffic is offloaded by the Internet. In this paper, we concentrated on an outdoor traffic model, where a user is connected to MME/S-GW through its nearest macro eNB. An MME serves a large number of eNBs under a tracking area. A user first sends its paging message to MME through its eNB before establishing a connection. The MME verifies the authenticator of the user with the help of a database called, Home Subscriber Server (HSS). After authentication an S1 link is established between eNB and the S-GW. The Serving Gateway (S-GW) route the internal traffic of the network. The Packet Data Network Gateway (P-GW) is responsible for routing traffic of external network under a trusted group of 3GPP where Evolved Packet Data Gateway (ePDG) does the work for the untrusted user group of 3GPP.

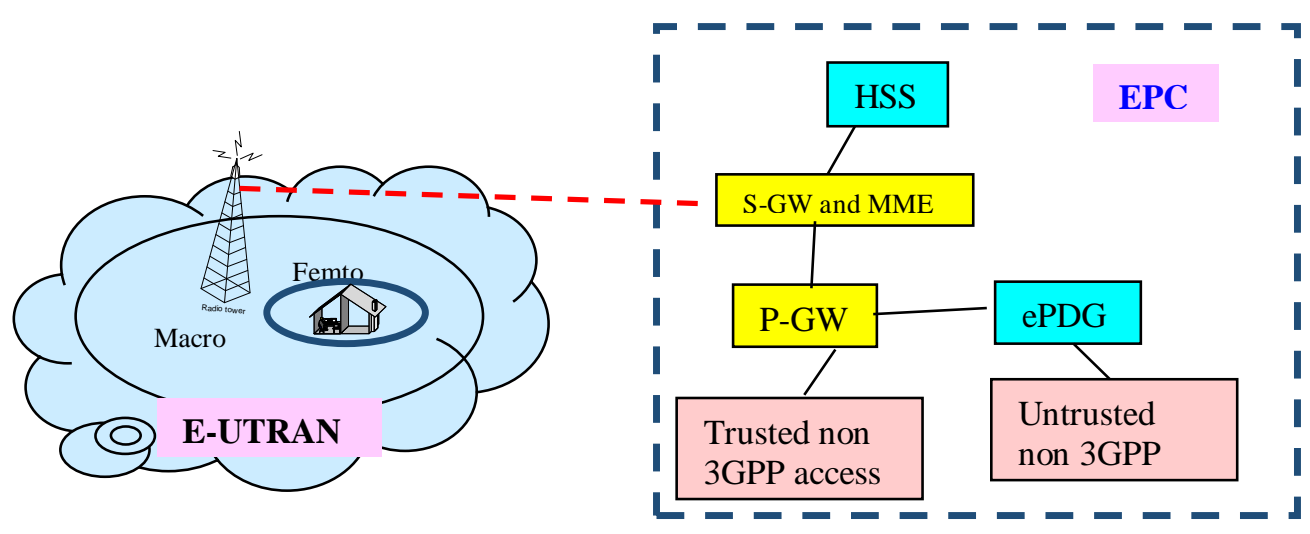

Fig.1: Physical architecture of LTE

In this paper, we propose a new radio network of LTE, where eNBs are connected by X2 links like the conventional network, and the entire traffic carried by the eNBs will be accumulated at a node nearest to the MME/S-GW. The nearest node is indicated by $n$ will be connected to the MME/S-GW by a high capacity S1 link as shown in fig.2. The combined traffic of the E-UTRAN is applied to EPC by the high capacity link has the capacity of C. Here all the eNBs can be considered as the sources and the noden where entire traffic is collected can be thought as the sink, hence maximum flow algorithm is applicable for such network.

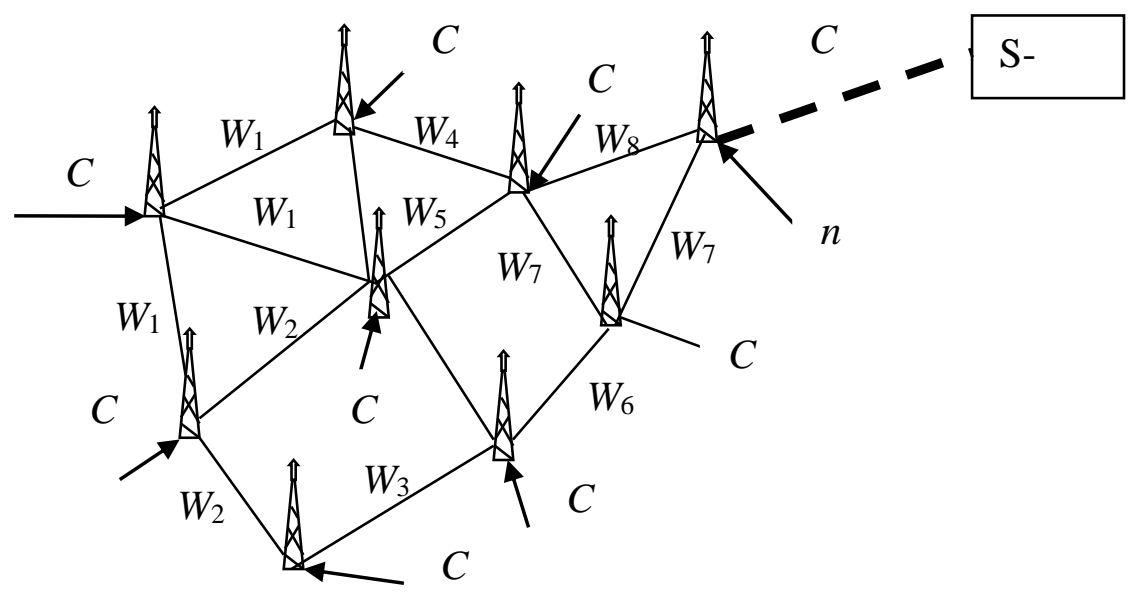

Fig.2:Universal Terrestrial Radio Access Network (UTRAN) of LTE 


\subsection{Traffic Model}

In fig. 2 if the offered traffic of $i$ theNB is $A_{i}$ then the aggregate offered traffic experienced on sink node will be,

$$
A=\sum_{i=1}^{n} A_{i}=\lambda_{T} t_{h}
$$

Where $\lambda_{T} \rightarrow$ arrival rate of resource block (RB) in RB/ms and $t_{h e} \rightarrow$ service time of an RB in ms. We know the duration of an RB is $0.5 \mathrm{~ms}$ and the number of symbols in $0.5 \mathrm{~ms}$ is 7 under normal cyclic prefix (CP). In QPSK each symbol carries 2 bits, therefore the number of bits per RB per subcarrier is $2 \times 7=14$ bits.

Now the bit rate/subcarrier $=14 / 0.5=28 \mathrm{Kbps}=0.028 \mathrm{Mbps}$.

The aggregate offered traffic, $A=\lambda_{T} t_{h}=0.5 t_{h}$

The node $n$ is anchored with the core switch hence node $n$ is considered as the sink of the maximum flow algorithm as found in [14-15]. The nodes $i=1,2,3, \ldots, n-1$ are the sources and we have only one node $n$ works as the sink, therefore the Ford-Fulkerson Algorithm in determination maximum flow needs some modification. In this paper, we propose the following modified algorithm where we add the superposition theorem with amaximum flow algorithm.

\subsection{Maximum Flow Algorithm with Superposition Theorem}

(a) Determine the weighted graph of the connected eNBs of the tracking area.

(b) Initialize all the edges with weight $W_{i j}$ between node $i$ and $j$ with the unit of Mbps.

(c) Take the node 1 as the source and make all the nodes of the network inactive (simply repeater) and the node $n$ works as the sink; provided node $n$ is connected to MME/S-GW.

(d) Determine maximum flow $Y_{1}$ from node 1 to $n$; where the offered data of node 1 is $C_{1}$ Mbps.

(e) Record all the used capacity $U_{i j}$ and remaining capacity $R_{i j}$ of each edge.

(f) If $Y_{1}<C_{1}$ then enhance the remaining capacity of links to secure $Y_{1} \geq C_{1}$.

(g) Repeat steps (c) to (e) for the node 2 with offered data rate of $C_{2}$ Mbps to secure maximum flow of $Y_{2} \geq C_{1}+C_{2}$.

(h) Repeat steps (c) to (e) to support the aggregate data flow of all sources to sink, $Y=\sum_{i=1}^{m} C_{i}$

Let the total flow is found as $C$ Mbps/subcarrier. Since the bit rate/subcarrier of a RB under QPSK is $0.028 \mathrm{Mbps}$ (as found before) therefore the number of RBs carried by the network simultaneously will be, $N=\left\lfloor\frac{C}{0.028}\right\rfloor$.

Let the field survey provides that each user on an average claims $m$ RB per call. If the capacity of the network is $N$ RBs then the number of channel of the network will be, $k=\left\lfloor\frac{N}{m}\right\rfloor$. 
Each user on the network generates average of $s$ calls/min, $m$ RB are used on each call and the duration of a call is $t_{h}$ min. The offered traffic per user in terms of RB is, $a=(s . m) t_{h}=s m t_{h}$ The total offered traffic, $A=a M=s m t_{h} M$.

\section{Example-1}

Let $M=200, m=3 \mathrm{RB} / \mathrm{call} / \mathrm{user}, t_{h}=1.5 \mathrm{mins}$ and $s=1 / 60 \mathrm{~min}$. If the current capacity of the network is $25 \mathrm{RB}$ then find the offered traffic and number of channel.

$A=\operatorname{smt}_{h} M=(1 / 60) \times 3 \times(1.5) 200=15$ Erls.

The number of channels, $k=\left\lfloor\frac{N}{m}\right\rfloor=\left\lfloor\frac{25}{3}\right\rfloor=8$

The blocking probability using Erlangs B formula of [16-17],

$$
B(A, k)=\frac{\frac{A^{k}}{k !}}{\sum_{i=0}^{k} \frac{A^{i}}{i !}}
$$

If $B(A, k)$ is weighted with the symbol error rate (SER) of QPSK and 16-QAM we get the relation between network traffic and SNR of the link (UE and eNB).

\section{Results}

In LTE two types of modulation schemes: QPSK and 16-QAM are used. When users are adjacent to eNB then they receive strong SNR and the system use 16-QAM (4 bits/symbol) again when users are at cell boundary experience huge fading then use QPSK scheme (2bits/symbol). Therefore throughput is higher when users are closed to eNB. In the section, we first consider the case of the QPSK scheme. The weighted graph of fig.3 is the interconnected eNBs, where each node corresponds to eNB and the edges are the wireless link among eNBs. Here we assume that each node offers the data of $2 \mathrm{Mbps} /$ subcarrier. This is not the typical value of the offered load of the LTE network, we only assume it to verify the profile of traffic parameters. The weight of each edge is the channel capacity (in Mbps) of that link. Here we use nine eNBs and found the maximum flow from the $i$ th node to sink node 9 (using Matlab 18) as shown in Table I. We consider, the number of users $M=50,100$ and 200, average RB /call $m=5$, average arrival rate per user: $(1 / 60) / \mathrm{min}$, and the modulation scheme of QPSK. Fig.4(a)-(c) show the variation of 'blocking probability' against 'offered average RB /call' taking 'average holding time' as a parameter. Here blocking probability increases with an increase in 'offered average RB /call', 'number of users', and 'holding time'. The rise of all curves is found exponential and mutually parallel. Actually 'offered average RB /call' $m$ is proportional to offered traffic at the same time inversely proportional to the number of channels hence fig. 4 shows the curves equivalent to offered traffic vs. blocking probability $(B)$ of Erlang's model. For all the curves of fig.4, we consider a network of low capacity i.e. $C=2.2 \mathrm{Gbps}$, therefore the blocking probability is found very high. In a practical network, capacity $C$ is thousands of times higher than the value used for fig. 4 hence practical blocking probability is very low. 
International Journal of Computer Science \& Information Technology (IJCSIT) Vol 12, No 4, August 2020

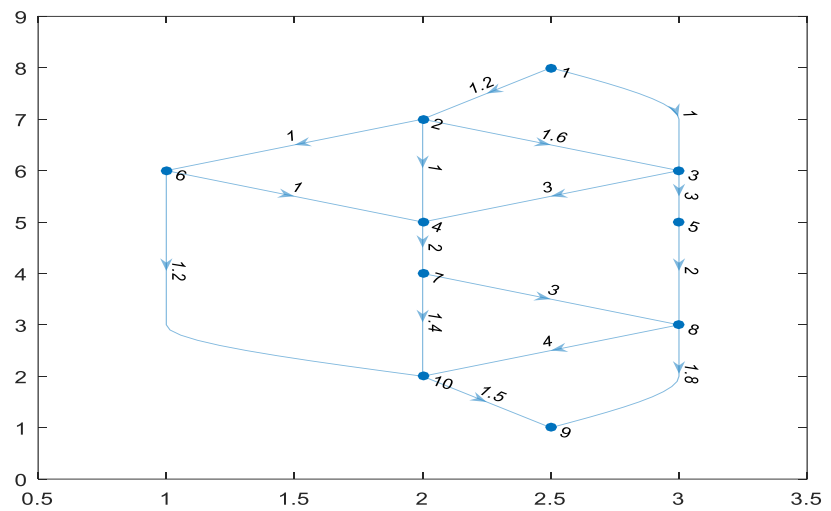

Fig.3 Equivalent weighted graph of interconnected eNBs of LTE

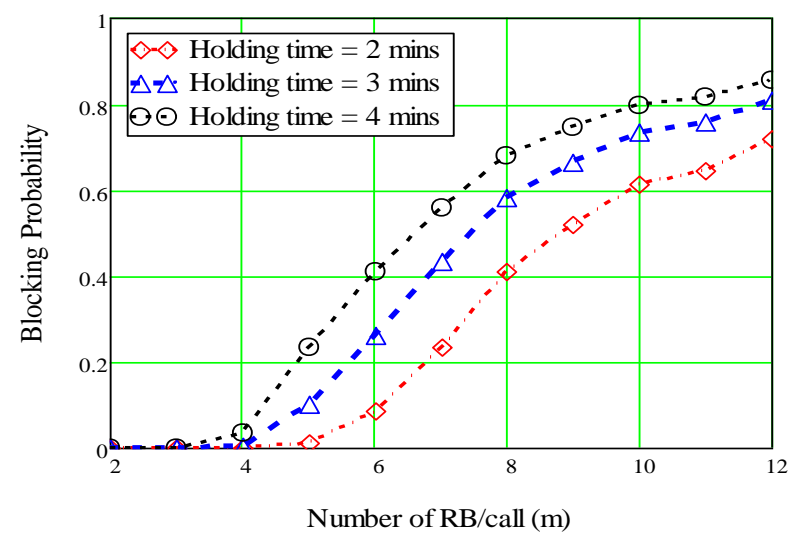

(a) $M=50$

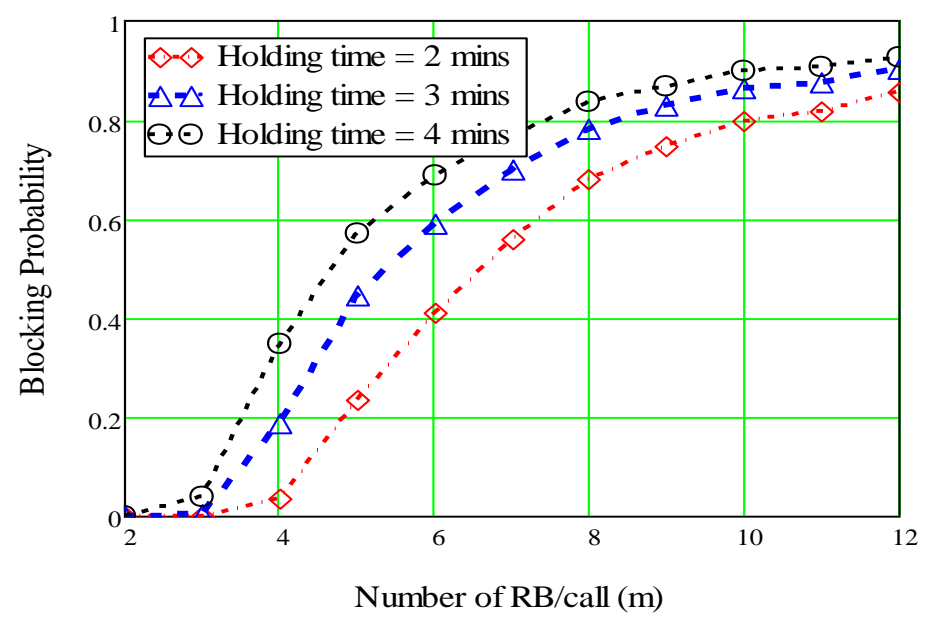

(b) $M=100$ 


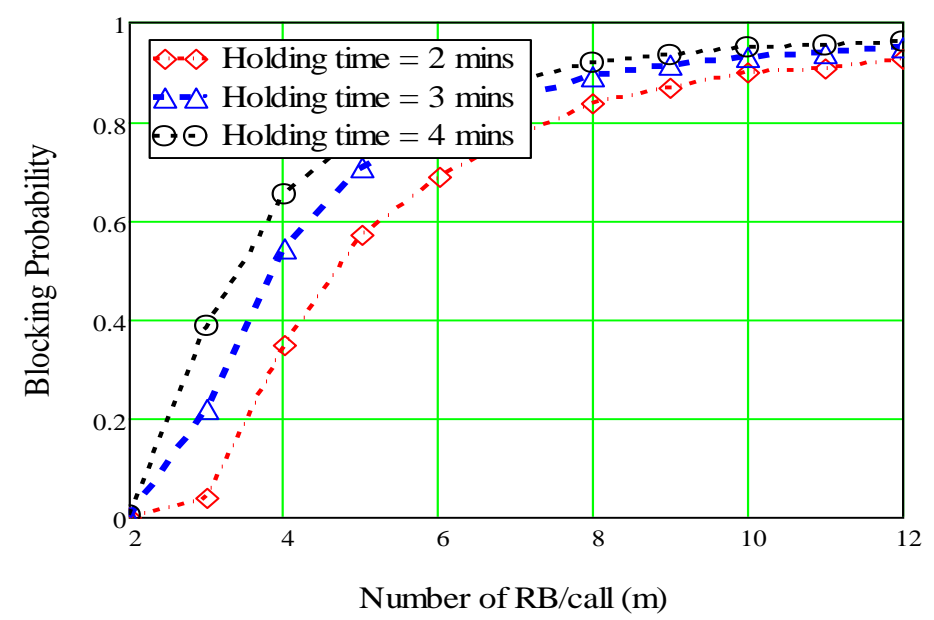

(c) $M=200$

Fig.4 Variation of offered RB/call $(m)$ against blocking probability

The variation of blocking probability against the 'simultaneous capacity of RB' taking 'average holding time' as a parameter is shown in fig.5. Three graphs are shown for $m=2,3$, and 4 . The 'simultaneous capacity of RB' is equivalent to the number of channels hence the curves of fig.5 are equivalent to the curve of 'number of channels' vs. blocking probability. The blocking probability decreases with an increase in the 'simultaneous capacity of RB' but reveres relation is found with 'average holding time'. The blocking probability also increases with an increase in $m$ is also visualized from fig.5 (a)-(c).

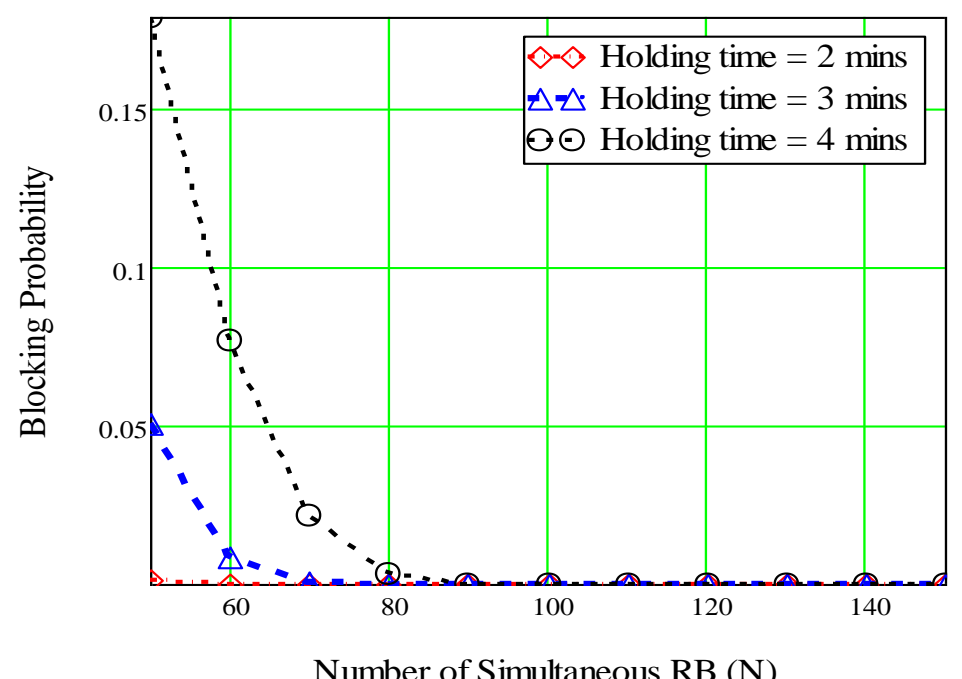

(a) $m=2$ 


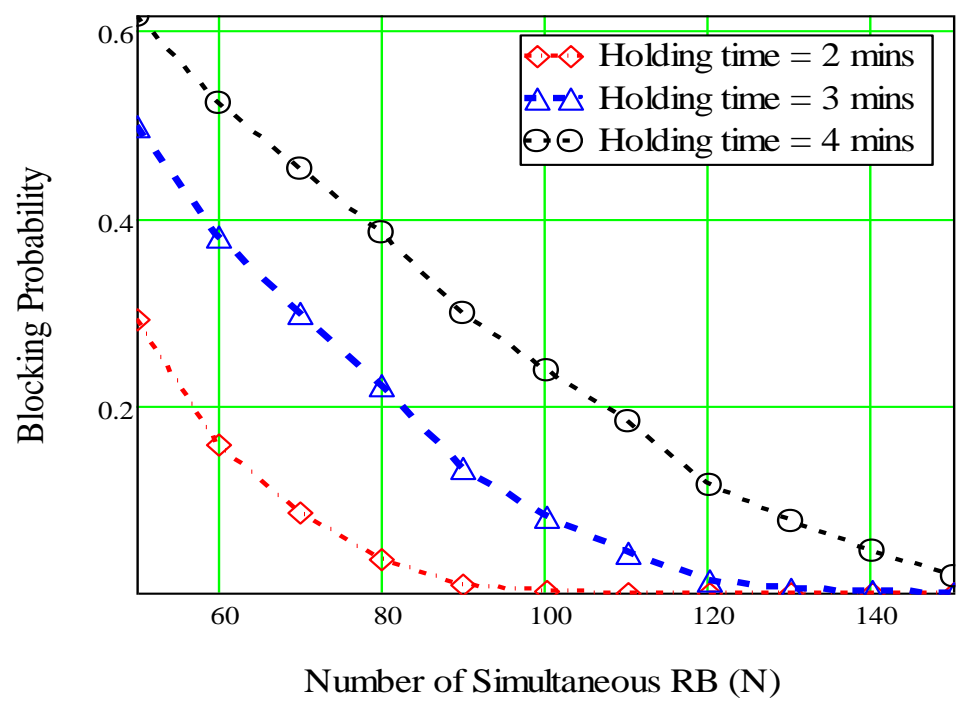

(b) $m=3$

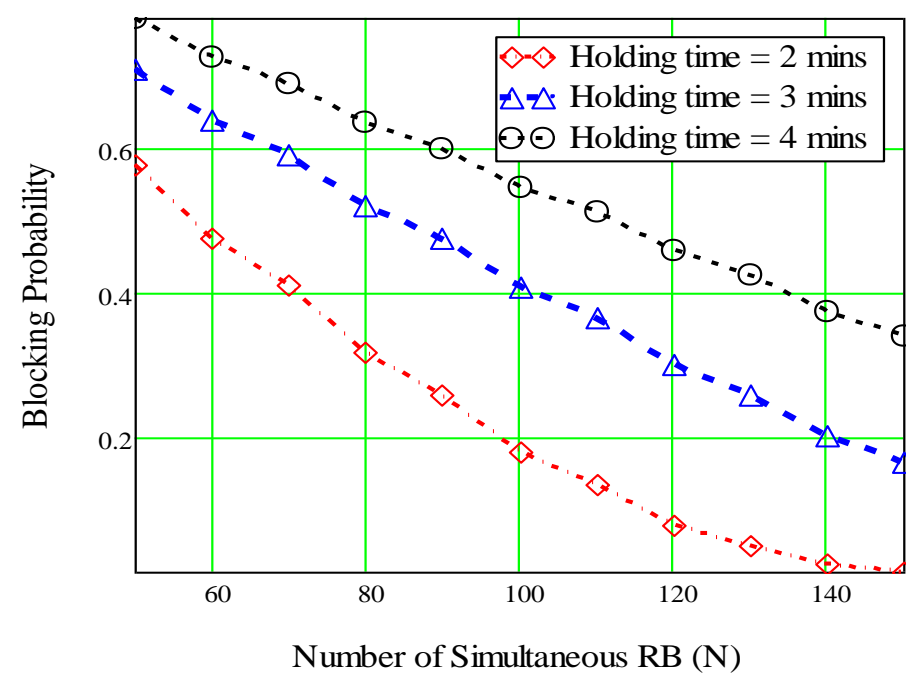

(c) $m=4$

Fig.5 Variation of the number of simultaneous $\mathrm{RB}(N)$ against blocking probability

Next, we consider a network of higher capacity as shown in fig.6; where we assume that each node offers the data of $4 \mathrm{Mbps} /$ subcarrier. Using the maximum flow algorithm, we got the flow of data from the $i$ th node to sink node 9 is shown in table II. Let the number of users is $M=50$, 100, and 200 and the capacity $C=9 \mathrm{Gbps}$. Now our aim is to observe the impact of incrementof capacity from source to sink like before. The blocking probability is found lower than the previous case as shown in fig.7 (a)-(c). 
International Journal of Computer Science \& Information Technology (IJCSIT) Vol 12, No 4, August 2020

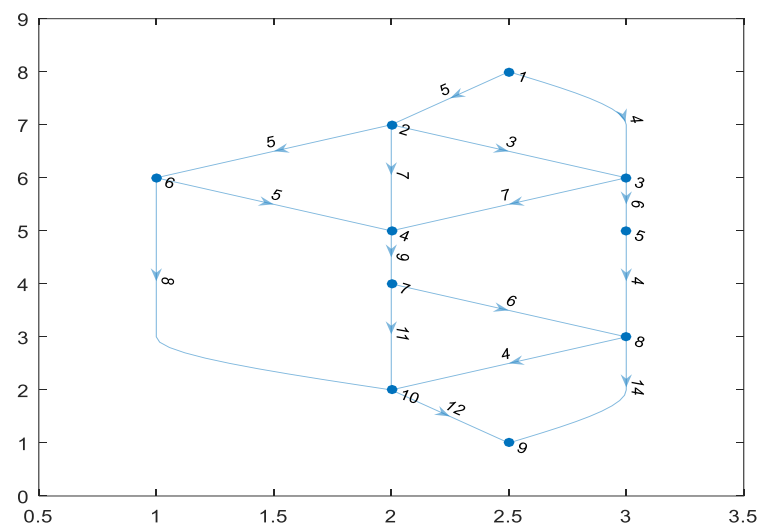

Fig.6 Equivalent weighted graph of interconnected eNBs of LTE of higher capacity

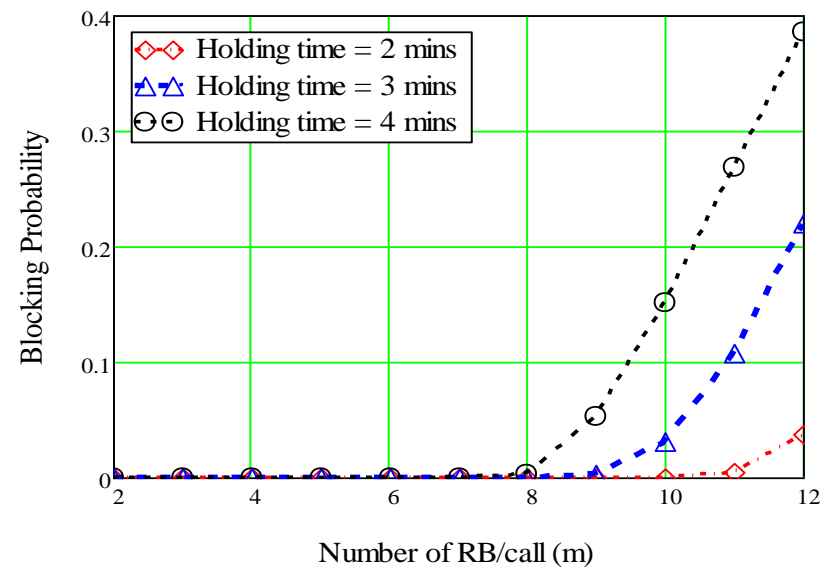

(a) $M=50$

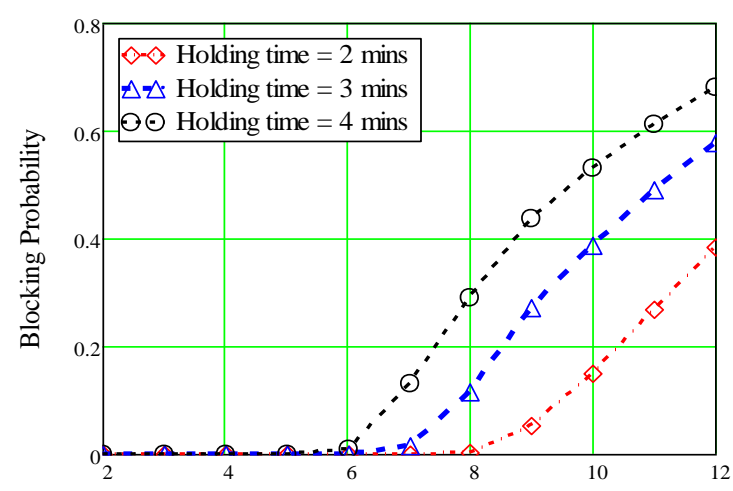

Number of RB/call (m)

(b) $M=100$ 
International Journal of Computer Science \& Information Technology (IJCSIT) Vol 12, No 4, August 2020

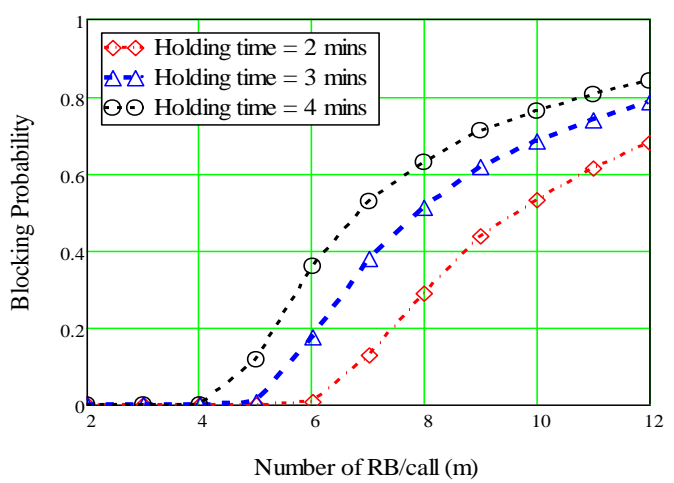

(c) $M=200$

Fig.7 Variation of offered RB/call $(m)$ against blocking probability under QPSK Table-I: Maximum flow per subcarrier

\begin{tabular}{|c|c|c|}
\hline Source & Sink & $\begin{array}{l}\text { Maximum flow } \\
\text { (Mbps/subcarrier) }\end{array}$ \\
\hline 1 & \multirow{8}{*}{9} & 2.2 \\
\hline 2 & & 3.3 \\
\hline 3 & & 3.3 \\
\hline 4 & & 2 \\
\hline 5 & & 2 \\
\hline 6 & & 2.2 \\
\hline 7 & & 3.3 \\
\hline 8 & & 3.3 \\
\hline
\end{tabular}

Table-II: Maximum flow per subcarrier

\begin{tabular}{|c|c|c|}
\hline Source & Sink & $\begin{array}{l}\text { Maximum flow } \\
\text { (Mbps/subcarrier) }\end{array}$ \\
\hline 1 & \multirow{8}{*}{9} & 9 \\
\hline 2 & & 15 \\
\hline 3 & & 11 \\
\hline 4 & & 9 \\
\hline 5 & & 4 \\
\hline 6 & & 13 \\
\hline 7 & & 17 \\
\hline 8 & & 18 \\
\hline
\end{tabular}

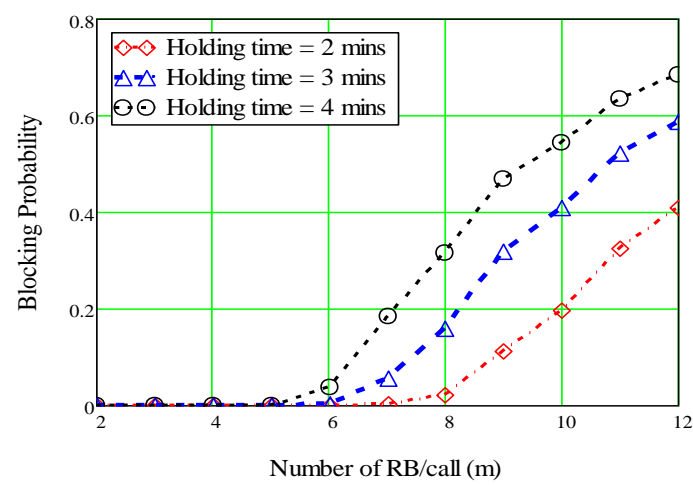

(a) $M=50$ 


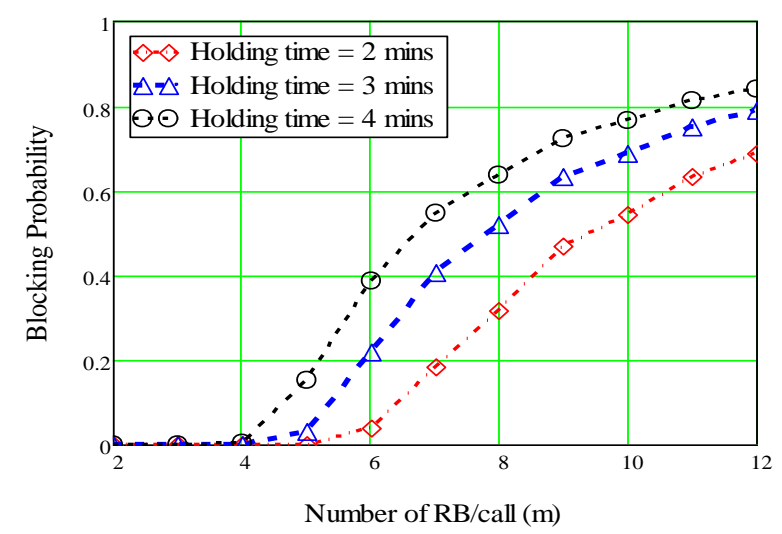

(b) $M=100$

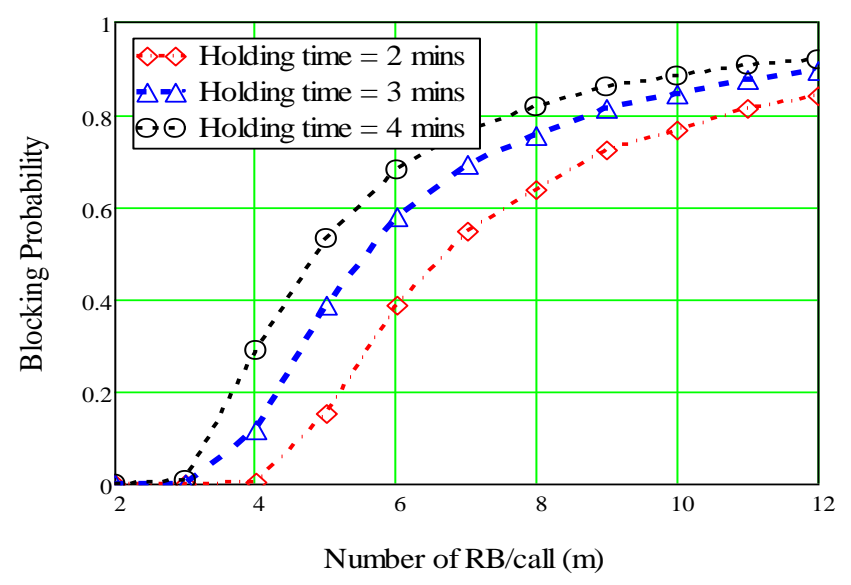

(c) $M=200$

Fig.8 Variation of RB/call against $B$ under 16-QAM

In 16-QAM the number of simultaneous RB carried by the network becomes, $N=\left\lfloor\frac{C}{0.056}\right\rfloor$ i.e. half of QPSK. Therefore the performance is deteriorated under 16-QAM visualized from fig. 8. Here $N$ is taken as the number of traffic channels of Erlang's model of the previous section, which is half compared to QPSK but throughput remains the same since each symbol of 16-QAM carries twice the bits of QPSK. Therefore the RR/call i.e. $m$ under 16-QAM is just half of QPSK to keep the same throughput hence performance remains the same like fig. 7. If we could relate the traffic model with SNR of link then we could get better performance under 16-QAM at higher SNR.

\section{Conclusions}

In this paper, we relate the simultaneous $\mathrm{RB}$, modulation scheme, and traffic parameters to measure the performance of the LTE network. The traffic model used here is $\mathrm{M} / \mathrm{M} / \mathrm{n}$, in future we will develop $\mathrm{M} / \mathrm{G} / \mathrm{n} / \mathrm{K}$ model applicable for $\mathrm{RB}$ of both fixed and variable length. Here we consider QPSK and 16-QAM under the awgn scheme. To get a more realistic result we have to consider small scale fading environments like Rayleigh or Nakagami- $m$ case. The entire result of the paper is analytical, in the future we will develop a simulation model with fading parameters. 


\section{REFERENCES}

[1] Jesmin Akhter, Abu Sayed Md. MostafizurRahaman, Md. Imdadul Islam, M. R. Amin' 'Traffic Modelling of Low Dense Femtocellular Network for Long Term Evolution,' Journal of Computer and Communications, pp.88-101, Vol.7, No.12, December 2019

[2] Ma Lin, Wei Shouming and Qiang Wei, 'A Novel Traffic Analysis Method For PoC over LTE Based on Retrial Calling Model,' 2011 6th International ICST Conference on Communications and Networking in China (CHINACOM), 17-19 Aug. 2011, pp.771-774, Harbin, China

[3] H. Hidayat, Al KautsarPermana, I. Ridwany, and Iskandar, 'Cell Capacity Prediction with Traffic Load Effect for Soft Frequency Reuse (SFR) Technique in LTE - A Network,' The 11th International Conference on Telecommunication Systems, Services, and Applications, 26-27 Oct. 2017, 26-27 October 2017, Lombok-Indonesia

[4] Haka, V. Aleksieva and H. Valchanov, 'Comparative Analysis of Traffic Prioritisation Algorithms by LTE Base Station Scheduler,' 2020 21st International Symposium on Electrical Apparatus \& Technologies (SIELA), pp. 1-4, 3-6 June 2020, Bourgas, Bulgaria

[5] M. Sahu, 'Delay Jitter Analysis for Uplink Traffic in LTE Systems,' 2019 11th International Conference on Communication Systems \& Networks (COMSNETS), pp. 504-506, 7-11 Jan. 2019, Bengaluru, India

[6] R. Liu, Q. Chen, G. Yu, G. Y. Li and Z. Ding, 'Resource Management in LTE-U Systems: Past, Present, and Future,' IEEE Open Journal of Vehicular Technology, vol. 1, pp. 1-17, Oct' 2020

[7] Bulbul Ahammad, Risala T. Khan and Md. Imdadul Islam, 'WLAN-LTE Integrated Traffic Model under Unlicensed Spectrum,' International Journal of Computer Science and Information Security (IJCSIS), vol. 17, no. 3, pp.85-100, March 2019

[8] Fatima Sapundzhi and MetodiPopstoilov, 'C\# implementation of the maximum flow problem,' 2019 27th National Conference with International Participation (TELECOM),pp. 62-65,30-31 Oct. 2019, Sofia, Bulgaria

[9] Y. Wang, J. Ling, S. Zhou, Y. Liu, W. Liao and B. Zhang, 'A Study on Rapid Incremental Maximum Flow Algorithm in Dynamic Network,' 2018 1st International Cognitive Cities Conference (IC3), pp. 7-11, 7-9 Aug. 2018, Okinawa, Japan

[10] Jiyang Dong, Wei Li, CongboCai, Zhong Chen, 'Draining Algorithm for the Maximum Flow Problem,' 2009 International Conference on Communications and Mobile Computing, pp.197-200, 68 Jan. 2009, Yunnan, China

[11] Ruipeng Bail ,HuiGuo, Zhenzhong Wang, Yanlong Zhang, Fan Zhang and Lei Chen, 'FPGA Interconnect Resources Test Based on A Improved Ford-Fulkerson Algorithm,' 2018 IEEE 4th Information Technology and Mechatronics Engineering Conference (ITOEC 2018), pp.251-258, 1416 Dec. 2018, Chongqing, China

[12] Jesmin Akhter, Md. Imdadul Islam, ASM M Rahaman and M R Amin, 'Performance Evaluation of Femtocell Based LTE Network under the Concept of Cross-layer Optimization,'International Journal of Computer Science and Information Security, pp. 52-60, vol. 14, no. 7, July 2016

[13] Jesmin Akhter, Md. Imdadul Islam, ASM M Rahaman and M R Amin, 'The MIMO Performance of LTE Network under Rayleigh Fading Environment,' International Journal of Computer Science and Information Security, pp. 88-94, vol. 14, no. 8, August 2016

[14] Lifeng Zhao and XiaowanMeng, 'An Improved Algorithm for Solving Maximum Flow Problem,' 2012 8th International Conference on Natural Computation (ICNC 2012), pp.1016-1018, 29-31 May 2012, Chongqing, China

[15] Bo Hong and Zhengyu He, 'An Asynchronous Multithreaded Algorithm for the Maximum Network Flow Problem with Nonblocking Global Relabeling Heuristic,' IEEE Transactions on Parallel and Distributed Systems, pp.1025-1033, vol. 22, no. 6, June 2011

[16] Ali Mustafa Elshawesh, Mohamed Abdulali, 'Dimensioning of Circuit Switched Networks by using Simulation Code based on Erlang (B) formula,' 2014 Global Summit on Computer \& Information Technology (GSCIT), pp. 1-5, 14-16 June 2014, Sousse, Tunisia

[17] James K. Tamgno, Mamadou Alpha Barry, Simplice E. Gnang, Claude Lishou, 'Estimating Number of Organs using Erlang's B \& C-Formulas,'2017 19th International Conference on Advanced Communication Technology (ICACT), pp.858-864, 19-22 Feb. 2017, Bongpyeong, South Korea 


\section{AUTHORS}

Birbahadur Khatri completed his B.Sc. in Computer Science and Engineering from Jahangirnagar University, Savar, Dhaka in 2015 and M.Sc. in the same discipline from the same University in 2016. He worked as a programming trainer in Green University of Bangladesh and as a software engineer at Newscred in Bangladesh from 2017 to 2018. Currently, he is working as a software engineer at Google in UK since 2019. He has excellent computer programming problem solving skill. He took part in many competitive programming contests both onsite

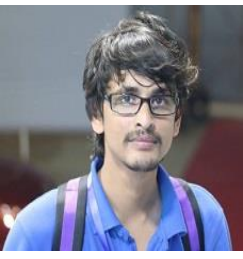
and online and has a very good contest rating in Codeforces. He is very enthusiastic at research work and his fields of interest are algorithm analysis and constructive algorithm design, wireless communication and machine learning.

Bulbul Ahammad completed his B.Sc. in Computer Science and Engineering from Jahangirnagar University, Savar, Dhaka in 2015 and M.Sc. in the same discipline from the same University in 2016. He worked as a lecturer at the department of Computer Science and Engineering in Daffodil International University from $1 \mathrm{st}$ January,2017 to 24th June 2019. He has been at the Department of Computer Science and Engineering as a lecturer in Jahangirnagar University since 25th June, 2019. He took part in many competitive programming contests and has a very good skill in

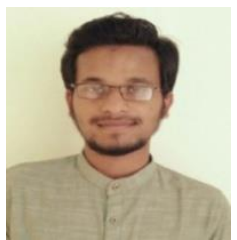
solving constructive computer programming problem. He has a great enthusiasm for innovative research work and his fields of research interest are machine learning, algorithm analysis and design, image processing and wireless communication.

Md. Mezbahul Islam received his B.Sc. (Honors) and M.Sc. in Computer Science and Engineering from Jahangirnagar University, Dhaka, Bangladesh in 2015 and 2017 respectively. He has been working as a faculty in the Department of Computer Science and Engineering, MawlanaBhashani Science and Technology University, Tangail, Bangladesh since April 2017. His research is focused in the fields of Image Processing, Pattern Recognition, Wireless Network and Machine Learning.

Rahmina Rubaiat completed her B.Sc. (Honors) and M.Sc. in Computer Science and Engineering from Jahangirnagar University, Dhaka, Bangladesh in 2015 and 2017 respectively. She worked as a faculty in the Department of Computer Science and Engineering, Brac University, Dhaka, Bangladesh since October 2015 to June 2019. Currently, she is working as a faculty member in the department of Computer Science and Engineering, MawlanaBhashani Science and Technology University, Tangail, Bangladesh since June 2019. Her research focused in the fields of Image Processing, Data Science, Pattern Recognition and Wireless Network.

Md. Imdadul Islam has completed his B.Sc. and M.Sc Engineering in Electrical and Electronic Engineering from Bangladesh University of Engineering and Technology, Dhaka, Bangladesh in 1993 and 1998 respectively and has completed his Ph.D degree from the Department of Computer Science and Engineering, Jahangirnagar University, Dhaka, Bangladesh in the field of network traffic in 2010. He is now working as a Professor at the Department of Computer Science and Engineering, Jahangirnagar University, Savar, Dhaka, Bangladesh. Previously, he worked as an

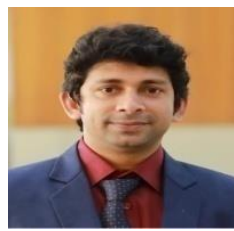

\section{(1)}

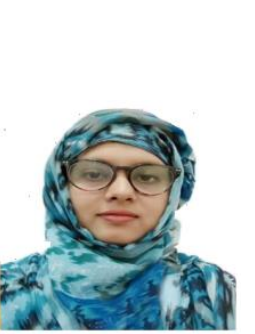
Assistant Engineer in Sheba Telecom (Pvt.) LTD (A joint venture company between Bangladesh and Malaysia, for Mobile cellular and WLL), from Sept.1994 to July 1996. Dr Islam has a very good field experience in installation and design of mobile cellular network, Radio Base Stations and Switching Centers for both mobile and WLL. His research field is network traffic, wireless communications, wavelet transform, adaptive filter theory, ANFIS, neural network, deep learning and machine learning. He has more than hundred and eighty research papers in national and international journals and conference proceedings. 